\title{
SOLVING THE ROUTING PROBLEM BY ANT COLONY OPTIMIZATION ALGORITHMS
}

\author{
Vladimir V. Zhikharevich ${ }^{1)}$, Nataliia A. Matsiuk ${ }^{2)}$, Sergey E. Ostapov ${ }^{1)}$ \\ 1) Yuriy Fedkovych National University of Chernivtsi, Chernivtsi, 2 Kotsyubinsky str., Chernivtsi, 58012, Ukraine, \\ vzhikhar@mail.ru, sergey.ostapov@gmail.com \\ ${ }^{2)}$ Bukovynian State Financial and Economics University, Chernivtsi, 1 M. Shterna str., Chernivtsi, 58002, Ukraine, \\ nataliya.matsiuk@gmail.com
}

\begin{abstract}
The use of ant colony optimization algorithms for solving the routing problem in a process of products delivery taking into account a city transport infrastructure has shown in this research. The vehicle routing problem belongs to NP-hard task and its solution requires significant computing resources. Therefore, it is recommended to use metaheuristic methods to solve such problems including ant colony optimization algorithms. Solution of the Vehicle Routing Problem will cause a decrease of enterprises non-productive resources consumption and will promote the increase of their efficiency and competitiveness. The test example, consisting of eight consumers of freight and two transportation means with unlimited load capacity, moving around the certain city, is used for the implementation of the model. It can be further refined by taking into account various parameters besides transport infrastructure, including limitations on carrying capacity, a number of vehicles an working hours, an amount of consumers' orders and a time for loading and unloading, etc. Copyright $\mathbb{C}$ Research Institute for Intelligent Computer Systems, 2014. All rights reserved.
\end{abstract}

Keywords: Ant Colony Optimization, Multiagent Modeling, Optimization, Vehicle Routing Problem, Transport Infrastructure.

\section{INTRODUCTION}

The vehicle routing problem (VRP) is a combinatorial optimization problem which is to find the shortest routes of vehicles fleet located in one or more depots, in the process of distribution of the products to the consumers. Thus, restrictions may be placed on the number and capacity of vehicles, the volume of customers' needs, delivery time etc.

Obviously, the routing problem is a generalized case of the traveling salesman problem (TSP) [14], when consumers' demand is accepted nonzero, and there are several agents that move. Such problems belong to the class of NP-hard ones, the solution of which requires significant computing resources and the time of optimal solution searching depends on the dimension of the problem exponentially. In this regard, while solving specific practical problems of this kind, when the dimension can reach 100 points or more application of the approximate methods is recommended [2]. Therefore, development and improvement of metaheuristic methods of solving combinatorial problems, which include, in particular, genetic algorithms (GA) and the method of ant colony optimization (ACO), is topical at the moment [5]. These methods work basing on similar processes that occur in living nature. Therefore, in some sources they can be named as "bioinspired algorithms" $[12,15]$.

Despite the fact that these meta-heuristic methods are combined in one research area entitled "natural computing", and allow finding close to optimal solutions of combinatorial problems in reasonable time, they differ both in the quality of solutions, and speed of work. Comparison of the efficiency of various metaheuristic methods for solving combinatorial problems was performed and presented in many research works. Although the higher efficiency of swarm intelligence methods (artificial bee colony and ant colony algorithms) compared to GA for solving NP-hard problems is proved in [10], it is indicated in [11] that TSP is more effectively solved by genetic and ant colony algorithms. We can assume that this statement is true for VRP due to the similarity of the two problems. This fact is confirmed by the authors of $[3,4]$ proving the effectiveness of genetic and ant colony algorithms and their combination in solving the routing problem.

However, to solve different variations of VRP, it is recommended to use different methods. For example, VRP with time windows is best solved by GA [4]. Instead, in solving VRP with limited 
carrying capacity, GA cannot compete with such a metaheuristic method as taboo search [3]. This leads to the trend of developing various combinations of existing methods, which has been observed in recent years. For example, in [3] a hybrid genetic algorithm for solving VRP with limited capacity is developed, based on the use of two populations where some individuals migrate periodically. It is noted in the above mentioned research work that a hybrid genetic algorithm for its efficiency can compete with the best method - the taboo search. The idea of using the multipopulation genetic algorithms is also developed in [1], but in this research the quality of genetic algorithm is enhanced by the method of ant colonies. Similarly, in [15] to solve TSP a hybrid algorithm is developed, the essence of which is to use sequential GA and ACO operators and the integration of genetic information into the process of building a road by the ACO agent. It is also proved that this algorithm allows finding better solutions than ACO, over the same time.

Examples of using ACO for solving the vehicle routing problem prove its effectiveness. In particular the paper [8], which describes the solving of school bus routing problem is a special case of VRP by ACO, allows reduce the total distance traveled by $8.3 \%$ and $21.4 \%$ respectively in the morning and in the afternoon. But most of them concern the applying of classical ACO for solution of different modifications of VRP, like VRP with stochastic demands [13], VRP with time windows [19], dynamic VRP [17]. At the same time all of them find near optimal solutions using Euclidean distances. However in the process of products delivery vehicles move on roads, so we need to obtain the solution of VRP with considering transport infrastructure rather than solution that is based on bird fly distances. Therefore this paper is devoted to description of ant colony optimization algorithm in optimizing the vehicle routing problem considering city transport infrastructure including the quality of roads and real driving situation.

\section{VEHICLE ROUTING PROBLEM}

The routing problem lies at the crossroads of two well-studied problems - the traveling salesman problem (TSP) and the bin packing problem (BPP) $[18,24]$. There are at least 7 types of routing problems [9], which differ in the degree of detailing. We consider the most common variation of the routing problem - the capacitated VRP (CVRP). It is an expanded version of the classic VRP and considers an additional parameter - the limited carrying capacity of the vehicle. Hereinafter CVRP will be mentioned as a conventional VRP.

The model routing problems includes the following parameters: $n$ - the number of customers (locations, to which the goods need to be delivered);

$K$ - carrying capacity of each vehicle;

$D=\left\{d_{i}\right\}_{i=1, n}-$ vector of demand (order volume) of customers;

$C=\left\{c_{i j}\right\}_{i, j=0, n}-$ matrix of values between fright transportation and consumers $i$ and $j$.

The essence of the routing problem is as follows. The same vehicles with limited carrying capacity $K$ deliver products from the depot $n_{0}$ to consumers $\overline{n_{1}, n_{n}}$. The challenge is to minimize the total cost of product delivery (or distances that all vehicles cover). That is, it is necessary to define a set of route of minimal total cost that would meet the following requirements:

- each route begins and ends at the depot;

- every consumer is included only in one of the routes as he may be served by only one vehicle;

- the total demand of each route consumers does not exceed the carrying capacity of the vehicle.

Considering all the introduced notations, VRP can be formalized as a weighted oriented graph $G=(N, A)$. The set of peaks $N$ includes the set of consumers $C$ (peaks $\overline{n_{1}, n_{n}}$ ) and the depot $n_{0}$. The set of graph edges is formed of the possible links between the peaks. Each edge $\left(n_{i}, n_{j}\right)$ corresponds to a certain cost of transportation between consumers $i$ and $j$, the cost of transportation from any point to the same point is, certainly, zero $c_{i i}=0$. Matrix of costs (distances) is symmetric when $c_{i j}=c_{j i}$. In the real world asymmetric matrix are more often, for example, due to the existence of one-way roads. The set of identical vehicles, characterized by a certain capacity $K$, will be denoted as $V$. Every consumer, in his turn, has a certain demand (the volume of its order) $d_{i}$. Only one value $X_{i j}^{v}$ serves as a variable which can take two values: 1 - if the vehicle moves from point $i$ to point $j, 0-$ in the other case.

The efficiency function of VRP is as follows [4]:

$$
\sum_{v \in V} \sum_{(i, j) \in A} c_{i j} X_{i j}^{v} \rightarrow \min
$$

The determined limitations are:

$$
\begin{aligned}
& \sum_{v \in V} \sum_{j \in N} X_{i j}^{v}=1 ; \forall i \in C, \\
& \sum_{i \in C} d_{i} \sum_{j \in N} X_{i j}^{v} \leq K ; \forall v \in V,
\end{aligned}
$$




$$
\begin{aligned}
& \sum_{j \in C} X_{0 j}^{v}=1 ; \forall v \in V, \\
& \sum_{i \in N} X_{i k}^{v}-\sum_{j \in N} X_{k j}^{v}=0 ; \forall k \in C, \forall v \in V, \\
& X_{i j}^{v} \in\{0,1\}, \forall(i, j) \in A, \forall v \in V .
\end{aligned}
$$

Limitation (2) means that every consumer should be served by only one vehicle. Inequality (3) establishes limitations on carrying capacity of vehicles. The total order of customers served by one vehicle shall not exceed its nominal capacity. Limitation (4) records that each vehicle can leave the depot once, and limitation (5) means that the number of vehicles that entered and left the consumer or depot should be the same.

This problem is solved in two stages:

1. Partition of the peaks set $N$ into $m$ subsets (routes);

2. Determining the sequence of visits to peaks in each route.

Solution of the routing problem can be represented as a graph, which is the union of $m$ oriented cycles of the original graph $G$ with a single point of intersection $n_{0}$ (Fig. 1).

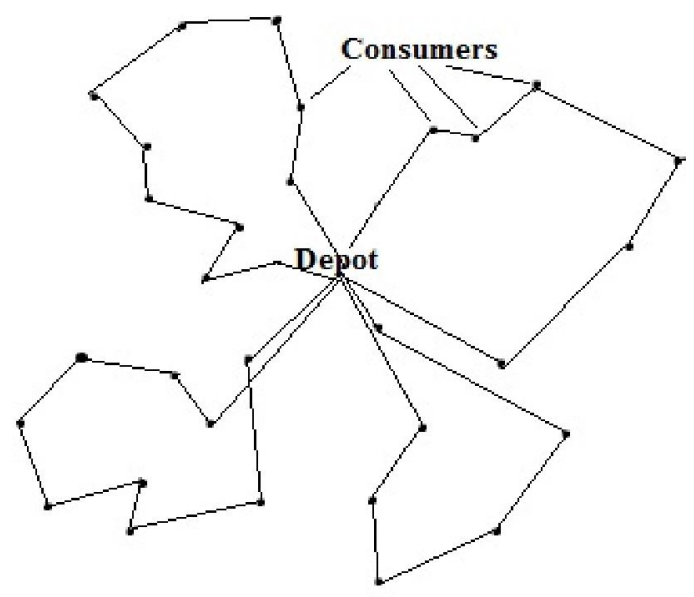

Fig. 1 - Presentation of the routing problem solution

Solving the routing problem describing real cases often requires more detail. Additional parameters included in the model may be asymmetric matrix of distances, several depots, heterogeneous vehicles, different time window for each consumer. Consideration of these factors makes solving this problem even more difficult. In this regard, the ant colony algorithm is one of the most common methods for solving this problem.

\section{ANT COLONY OPTIMIZATION ALGORITHM}

The ant colony algorithm imitates the behavior of an ant colony in the process of finding the shortest path from the anthill to a food source [6]. The basic principle, which is possible thanks to intelligent behavior of the colony, according to S.D. Shtovba's definition, is the principle of self-organization as a dynamic set of mechanisms for the achievement of the global objectives as a result of low-level interaction between its elements [21]. This interaction means that the elements of the system use only local information, while any centralized influence is eliminated. In such cases, swarm intelligence is considered as a different type of cooperative behavior. A colony of ants is essentially a multi-agent system, the agents of which interact according to basic rules [7]. Thus, despite the primitive behavior of agents, the behavior of the system is extremely reasonable and close to optimal [21].

Ant colony algorithms are based on the use of artificial ants similar to the behavior of real ants. The interaction between the ants in nature is performed by using direct and indirect methods of information transmission. The direct way is to share a meal, as well as visual and chemical contacts. The indirect method is based on the use of some chemical substance (pheromone) by the ants trapped in a certain area, which remains as a trace after the movement of other ants. This type of interaction is distributed in time and is called stigmergy [21]. Stigmergy work as follows. In search of food an ant moves randomly, leaving a constant amount of pheromone. When another ant encounters this trace, it must decide whether to move along it or not. If it decides to move along it, its own pheromone strengthens the existing trail, which increases the probability that the next ants will choose this path. Thus, the more ants moving a certain way, the more attractive it becomes for other ants. In addition, the ant that uses a shorter route, quickly returns to the anthill, and thus leaves its pheromone twice. Thus, the pheromone is accumulated on shorter routes. In addition, the pheromone evaporates over time, making detection of less desirable routes more difficult, and therefore reduces their use. But nevertheless, the random selection of ways allows the ant colony to identify alternative routes and ensures successful bypass of obstacles interrupting the route. This creates conditions for adaptability of the colony's behavior.

Ant colony algorithms were first used to solve the traveling salesman problem, which is a kind of test ground for the algorithms for combinatorial optimization. Taking into account the similarity of the traveling salesman problem and the routing one, the ant colony algorithms have been successfully applied in this area [2].

Self-organization of the ant colony in nature is possible due to the following component: accident, 
repetition, positive feedback, negative feedback [21]. Repetition of interaction of ants in case of solving the routing problem is implemented through simultaneous iterative search for transport route by several ants that act autonomously. Positive feedback is embodied in the rule: the more pheromone a certain edge count contains, the greater the probability of its inclusion in the route is. However, the exclusive use of positive feedback algorithm can lead it to a local minimum. Therefore the negative feedback is used, which consists in evaporation of pheromone. Due to this property the colony is able to adapt to changing external conditions, for example, to find alternative routes in case of obstacles arising on the way from the anthill to a food source.

While solving the routing problem using ant colony algorithms, each vehicle is simulated by a separate ant, and the route is being built gradually until all the consumers are included in it. Each ant starts its way to the depot, and it chooses every next point basing on the list of unvisited points and carrying capacity of a vehicle. Every single passing of all points by the ant is provided by its "memory" which contains information about the list of points already passed, the so-called list of prohibitions. The list of unvisited points of the $k$-th ant, which is at the point denoted $i$ will be denoted as $J_{i, k}$.

Besides memory, ants have "eyesight" that is embodied in the parameter of "visibility". Visibility is a value inverse to that of the distance between points. The greater visibility, the more the ant's the desire to travel to the point $j$ from the point $i$.

Another property taken from a colony of ants and included in the ant colony algorithm is "smell". Ants feel the pheromone trail left by other ants that embodies their experience of passing points. The amount of pheromone on the edge $(i, j)$ at the moment of time $t$ will be designated as $\tau_{i j}(t)$.

The ant decides whether to move from point $i$ to point $j$ on the $t$-th iteration due to probabilistic rule [21]:

$$
\left\{\begin{array}{l}
P_{i j, k}(t)=\frac{\left[\tau_{i j}(t)\right]^{\alpha} \cdot\left[\eta_{i j}\right]^{\beta}}{\sum_{l \in J_{l, k}}\left[\tau_{i l}(t)\right]^{\alpha} \cdot\left[\eta_{i l}\right]^{\beta}}, \text { if } j \in J_{i, k}, \\
P_{i j, k}(t)=0, \text { if } j \notin J_{i, k},
\end{array}\right.
$$

where $\alpha$ and $\beta$-parameters that are defined experimentally and determine the amount of weight of pheromone significance and visibility when choosing a route. If $\alpha=0$, the next route is chosen, and then this algorithm becomes similar to the greedy algorithm of the classic optimization, and if $\beta=0$, only pheromone trail is important that leads to quick finding of the local optimum, i.e. premature convergence of the algorithm.

The pheromone trail laid by the $k$-th ant on the edge $(i, j)$, is defined as:

$$
\Delta \tau_{i j, k}(t)=\left\{\begin{array}{l}
\frac{Q}{L_{k}(t)}, \text { if }(i, j) \in T_{k}(t), \\
0, \text { if }(i, j) \notin T_{k}(t),
\end{array}\right.
$$

where $T_{k}(t)$ - the route that the $k$-th ant passed on the $t$-th iteration length $L_{k}(t) ; Q$ - adjustable parameter the value of which is of the same order as the length of the optimal route.

Evaporating, the pheromone is restored by the following rule:

$$
\tau_{i j}(t+1)=(1-p) \cdot \tau_{i j}(t)+\Delta \tau_{i j}(t),
$$

where $p \in[0,1]$ - the pheromone evaporating index, $\Delta \tau_{i j}(t)=\sum_{k=1}^{m} \Delta \tau_{i j, k}(t), m-$ the number of ants in the colony.

At the beginning of the algorithm the amount of the pheromone is assumed to be a small positive number. The number of ants through the algorithm duration remains constant. Typically, the number of ants equals to the number of points, and each ant starts its way from its own point. In our case the number of ants equals to the number of network structure nodes of city roads.

The ant returns to the depot, when all consumers are visited or when it reaches critical carrying capacity. Moreover, at first the algorithm builds the complete route for the first ants, and when it returns to the depot, begins to build the route for the second one and so on. This occurs until the specified number of ants with known carrying capacity visits all the consumers. Stopping criterion of algorithm is the situation, when the "ants moving on the same optimum path", i.e. the paths of the ants between points are the same.

Computation speed of the algorithm can be estimated by the number of iterative cycles $K \sim \mathrm{N} / \mathrm{p}$, where $\mathrm{N}$ - number of the network structure nodes, $\mathrm{p}$ - evaporation rate of pheromone. So the computation speed is directly proportional to the number of the network nodes of the transport infrastructure and inversely proportional to the evaporation rate of pheromone.

\section{RESULTS OF THE COMPUTATIONAL EXPERIMENT}

To implement the ant colony algorithm for solving the routing problem a number of assumptions were made: 
- the number of destinations located randomly, equals 8 ;

- all the goods are distributed in two vehicles;

- the carrying capacity of vehicles is sufficient enough to deliver all goods in one route;

- the total customers' order does not exceed the nominal carrying capacity of two vehicles;

- the timetable, except for the time of transportation, are equal to zero; this is the time for unloading and for lunch (the time required for unloading products can be taken into account by adding some averaged values of this time to the total time, multiplied by the number of points).

These simplifications do not go beyond the primary objective of our research, which consists in demonstration of ant colony algorithms to solve the routing problem.

Implementation of ant colony algorithm for solving the routing problem in this case primarily involves representing transportation infrastructure in some network form. The process of this presentation is schematically shown in Figures 2-5.

Fig. 5 shows the final form of a fragment of road infrastructure of the city. Thus, the problem of optimizing some fright transportation is to find the set of minimum distances between the given network nodes.

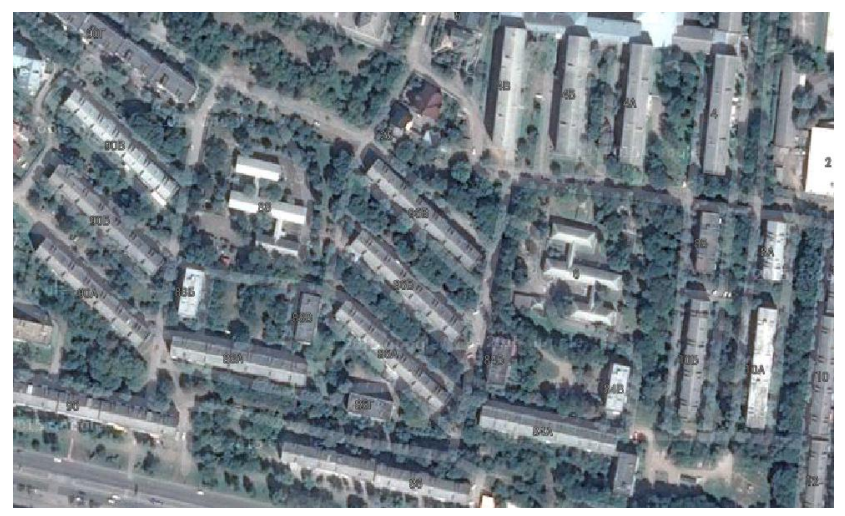

Fig. 2 - Satellite photo fragment of city infrastructure

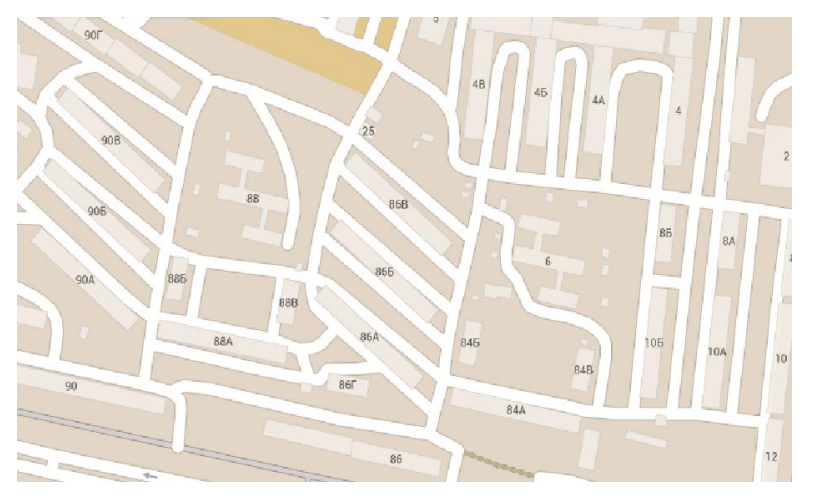

Fig. 3 - Schematic representation of a fragment of city infrastructure

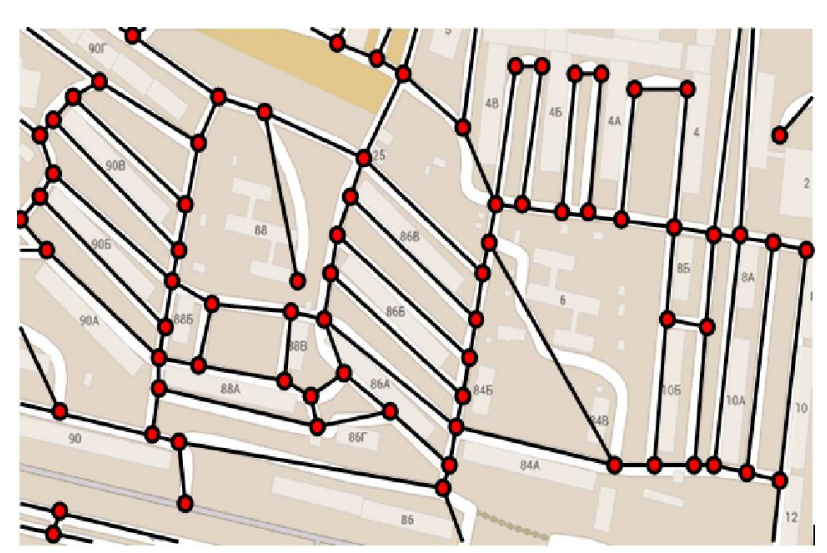

Fig. 4 - Example of forming a network structure of city roads

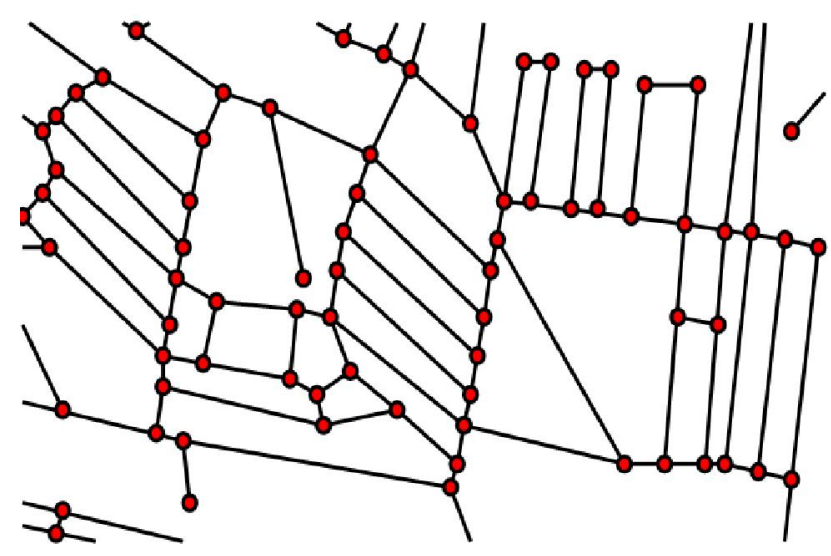

Fig. 5 -View of network structure of city roads

Besides consideration of distances between nodes, in real situations, the information that minimizes the time spent to overcome this distance is more useful. It is defined by the following factors:

1) the quality of the road surface;

2) the availability and regularity of traffic lights switch;

3) the number of lanes (road capacity);

4) the number of third-party machines in specific time periods (formation of traffic jams);

5) the average time taken to make a turn (Fig. 6).

In addition to these, we can mention a number of factors that depend on many subjective and objective reasons (season, time of day, etc.).

In the final case, all these factors can be reduced to the average time to overcome the specific sections of the road, depending on the average speed on this section and its length. Besides, sometimes on some roads there can be a one-way part (Fig. 6 d), which clearly affects the trajectory of the vehicle.

In our work, to analyze the efficiency of ant colony algorithm in solving the fright transportation problem, a model of a fragment of road infrastructure of the city and points, to which the goods need to be delivered, was generated (Fig. 7). The length of the fragment $-10 \mathrm{~km}$, the width -5 
$\mathrm{km}$. In addition, there are three types of roads with different average speeds assigned for them - 10, 20 and $30 \mathrm{~km} / \mathrm{h}$, highlighted respectively, from light gray to black. The average time taken for rightturning models is $10 \mathrm{sec}$, and for left-turning ones $20 \mathrm{sec}$. Also, some parts of roads are one-way roads (this fact is not shown in the figures to avoid the image).
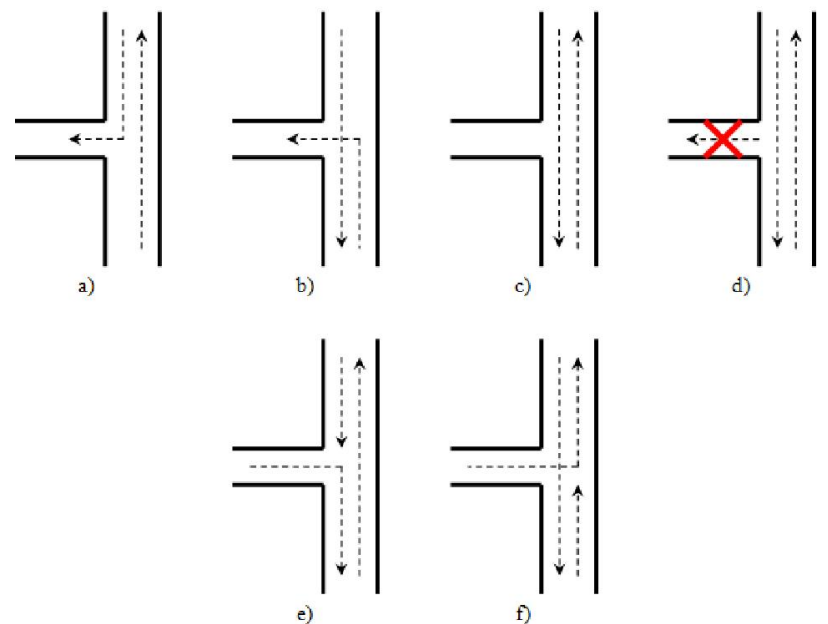

Fig. 6 - Examples of different variants of traffic situations affecting the average time of turn

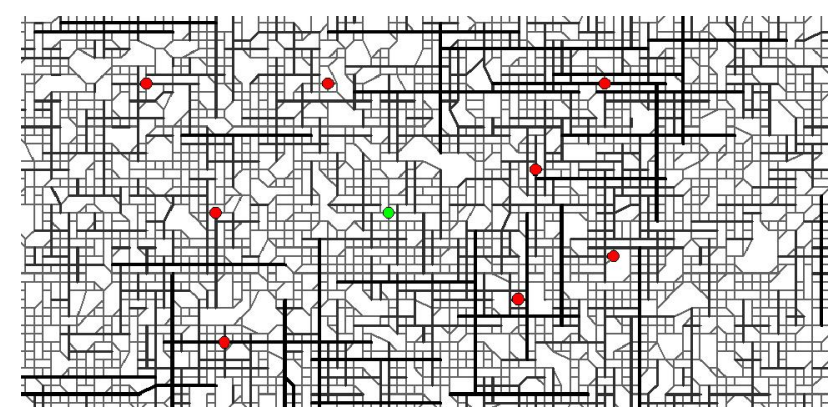

Fig. 7 - Example of the generated road infrastructure of the city fragment. Points are product consumers (green circle - depot, red ones - consumers)

All nodes of the network structure of roads have their indices, and the indices and types of roads to the closest neighboring nodes. As it was mentioned above, the routing problem is solved in two stages. The first stage lies in the partition of the set of peaks into subsets (routes), and the second one - in determining the sequence of peaks visiting in each route. Destinations according to the generated model are distributed between two vehicles in the most optimal way, and the path of a vehicle is reduced to enumeration of the order of nodes. Fig. 8 shows an example of paths of two vehicles under the condition of minimal path length. The left path has a total length of $12.25 \mathrm{~km}$; the elapsed time -1.05 hours, and the right one $-10.45 \mathrm{~km}$ with elapsed time 0.85 hours.

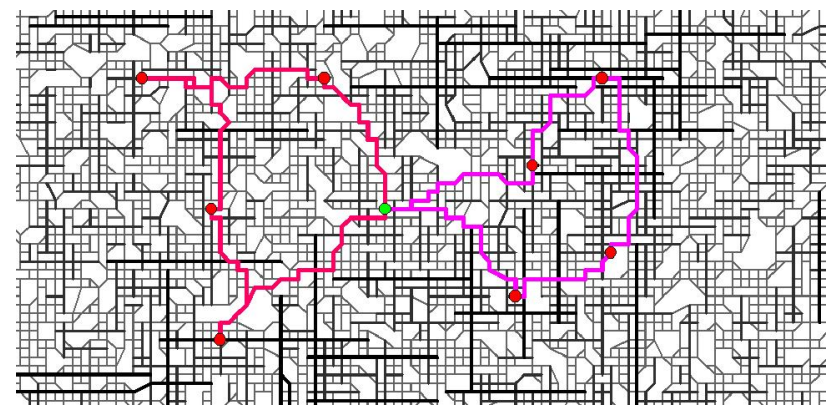

Fig. 8 - Example of two vehicle's paths with minimal path length (left - counterclockwise, right - clockwise)

At the same time, the minimal way in real life is not necessarily the fastest due to the influence of the aforementioned factors, such as: quality of paving, traffic jams, etc. In terms of minimizing the cost of fuel certainly it is more important to find the shortest route for vehicles. However, the loss of time due to their standstill in traffic jams or at traffic lights can lead to failure of a product delivery plan that will result in the loss of image of the company, which is more important in long-term relationships with partners. However, going by the roads with low quality of paving could lead to additional costs associated with unscheduled repairs of vehicles. Therefore, in our view, it is more appropriate in such circumstances to optimize the path of vehicular traffic by the time, but not by length. Fig. 9 shows an example of paths of two vehicles under the condition of minimal time of transportation.

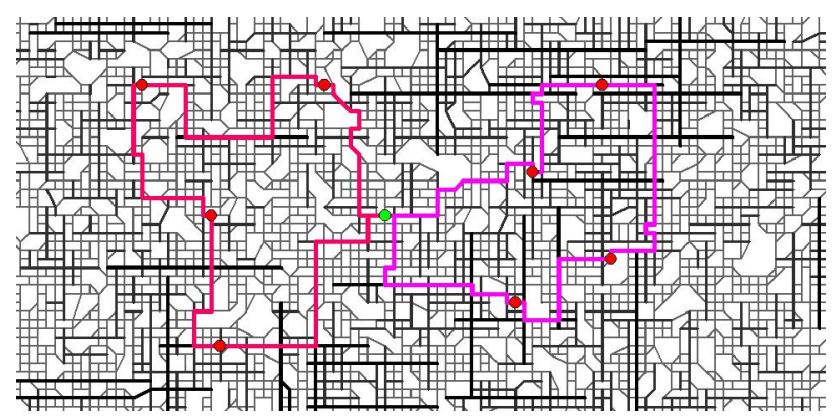

Fig. 9 - Example of two vehicle's paths under the condition of minimal transportation time (left counterclockwise, right - clockwise)

The left path has a total length of $13.62 \mathrm{~km}$ with the time 0.79 hours, while the right one $-12.34 \mathrm{~km}$ with the time of 0.65 hours. It is obvious that in the second case, despite the greater distance passed by vehicles, time is less.

\section{CONCLUSION}

Thus, the fundamental possibility of using the ant colony algorithms to solve the routing problem was demonstrated in this research work. Optimization of routes of vehicles during the distribution of the 
products will reduce unproductive expenditures that will positively affect the profitability and competitiveness of enterprises. To implement the model the test example was used with eight pointsconsumers of goods and two vehicles with unlimited carrying capacity moving on the roads of a certain city. The model can be further detailed by taking into account various parameters, including limitations on carrying capacity, the number of vehicles, working hours, the volume of orders of consumers, time for loading and unloading, etc. In this case, the considered routing problem becomes a combinatorial optimization problem with many limitations, which should be solved by the hybrid methods. For example, it may be a combination of genetic and ant colony algorithms. Taking into consideration the set of parameters and their optimization will be carried out by means of genetic algorithm while the ant colony algorithm will be used as an auxiliary one to find minimal way. This is the prospect for our further research.

\section{REFERENCES}

[1] V. V. Beglyarov, A. N. Beryoza, A. S. Storozhenko, "Hybrid ant multipopulation genetic algorithm," Herald of YuFU. Technical Science, no. 7, pp. 39-45, 2010. (in Russian)

[2] J. E. Bell, P. R. McMullen, "Ant colony optimization techniques for the vehicle routing problem," Advanced Engineering Informatics, no. 18, pp. 41-48, 2004.

[3] J. Berger, M. Barkaoui, "A new hybrid genetic algorithm for the capacitated vehicle routing problem," Journal of the Operational Research Society, vol. 41, issue 2, pp. 179-194, 2003.

[4] A. S. Bjarnadottir, Solving the Vehicle Routing Problem with Genetic Algorithms, Technical University of Denmark, 2004, 127 p.

[5] C. Blum, A. Roli, "Metaheuristics in combinatorial optimization: overview and conceptual comparison," ACM Computing Surveys, vol. 35, issue 3, pp. 268-308, 2003.

[6] M. Dorigo, "Ant colonies for the traveling salesman problem," Biosystems, vol. 43, pp. 73-81, 1997.

[7] M. Dorigo, G. Di Caro, "Ant algorithms for discrete optimization," Artificial Life, vol. 5, issue 3, pp. 137-172, 1999.

[8] J. S. Arias-Rojas, J. F. Jiménez, J. R. MontoyaTorres, "Solving of school bus routing problem by ant colony optimization," Revista EIA, vol. 17, pp. 193-208, 2012.

[9] E. A. Kochegurova, Yu. A. Martyinova, "Optimizing compilation of public transport routes when creating an automated decision support system," Herald of the Tomsk Polytechnic University, vol. 323, issue 5, pp. 79-84, 2013. (in Russian)

[10] V. M. Kureychik, A. A. Kazharov, "The using bee algorithms for combinatorial problems," Artificial Intelligence, issue 3, pp. 583-589, 2010. (in Russian)

[11] V. M. Kureychik, A. A. Kazharov, "The using of swarm intelligence in solving of the NP-hard problems," Herald of YuFU. Technical Science, no. 7, pp. 30-36, 2011. (in Russian)

[12] V.V. Kureychik, V.M. Kureychik, S. I. Rodzin, "The concept of evolutionary computation inspired by natural computing," Herald of YuFU. Technical Science, Special issue "Intelligent CAD", Taganrog, Publishing House TTI YuFU, vol. 4, issue 93, 256 p., 2009. (in Russian)

[13] M. Manfrin, Ant Colony Optimization for the Vehicle Routing Problem, DEA defense at ULB, Brussels, 2004.

[14] Mao-xiang, L., Hu, Si-ji, "Study on the optimization of physical distribution routing problem by using hybrid genetic algorithm," China Journal of Management Science, vol. 10, issue 5, pp. 51-56, 2002.

[15] A. V. Martyinov, V. M. Kureychik, "Hybrid algorithm for solving the traveling salesman problem," Herald of YuFU. Technical Science, vol. 4, issue 165, pp. 36-44, 2015. (in Russian)

[16] N. Matsiuk, "Solution of travelling salesman problem for wholesale outlays considering repeated visitation of certain sales outlets," MEST Journal, Belgrade : MESTE, vol. 3, issue 1, pp. 120-126, 2015.

[17] R. Montemanni, L. M. Gambardella, A. E. Rizzoli, A. V. Donati, "Ant colony system for a dynamic vehicle routing problem," Journal of Combinatorial Optimization, vol. 10, pp. 327-343, 2005.

[18] Li, N., Wang, Sh., Li, Yu, "A hybrid approach of GA and ACO for VRP," Journal of Computational Information Systems, vol. 7, issue 13, pp. 4939-4946, 2011.

[19] T. Pellonperä, Ant Colony Optimization and the Vehicle Routing Problem, University of Tampere, 2014, $51 \mathrm{p}$.

[20] R. Ruiz, C. Maroto, "A decision support system for a real vehicle routing problem," European Journal of Operational Research, vol. 153, issue 3, pp. 593-606, 2004.

[21] S. D. Shtovba, "Ant algorithms, exponenta pro," Math Applications, no. 4, pp. 70-75, 2003. 
[22] Shuoben Bi, Xueshi Dong, Yan Ma, "The design and analysis of TSP problem based on genetic algorithm and ant colony algorithm," International Journal of Education and Management Engineering, vol. 2, issue 9, pp. 56-60, 2012.

[23] W. Zhong, Using Traveling Salesman Problem Algorithms to Determine Multiple Sequence Alignment Orders, Master Thesis, The University of Georgia, Athens, Georgia, 2003, $66 \mathrm{p}$.

[24] K. Zoulel, F. C. Besma, K. Mekki, “TSP based evolutionary optimization approach for the vehicle routing problem," in Proceedings of the 2nd Mediterranean Conference on Intelligent Systems and Automation, 23-25 March 2009, pp. 373-376.

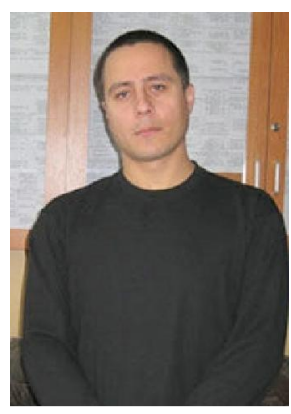

\section{Vladimir}

Zhikharevich, $P h D$. of physical and mathematical sciences, associate professor of the Department of Computer Systems Software, Yuriy Fedkovych Chernivtsi National University.

Areas of scientific interests: Application problems in the theory of cellular automata, the

theory of self-organizing systems (synergy).

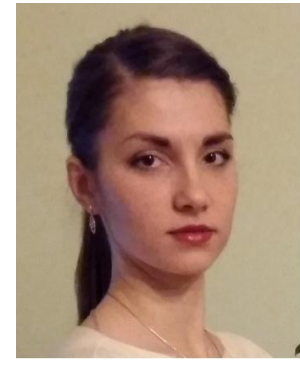

ling.

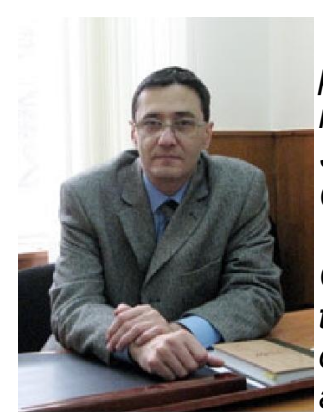

neering tasks.
Matsiuk Nataliia, PhD student, assistant of the Department of Enterprise Economy, Bukovina State Finance and Economics University.

Areas of scientific interests: optimization of business processses of wholesale enterprises, cellular automata mode-

Sergey Ostapov, Dr. Sci., professor, head of the Department of Computer Systems Software, Yuriy Fedkovych Chernivtsi National University.

Areas of scientific interests: Cellular automata modeling, intelligent analysis and processing of information, components of artificial intelligence in engi- 